\title{
Enhancing the Power of Understanding
}

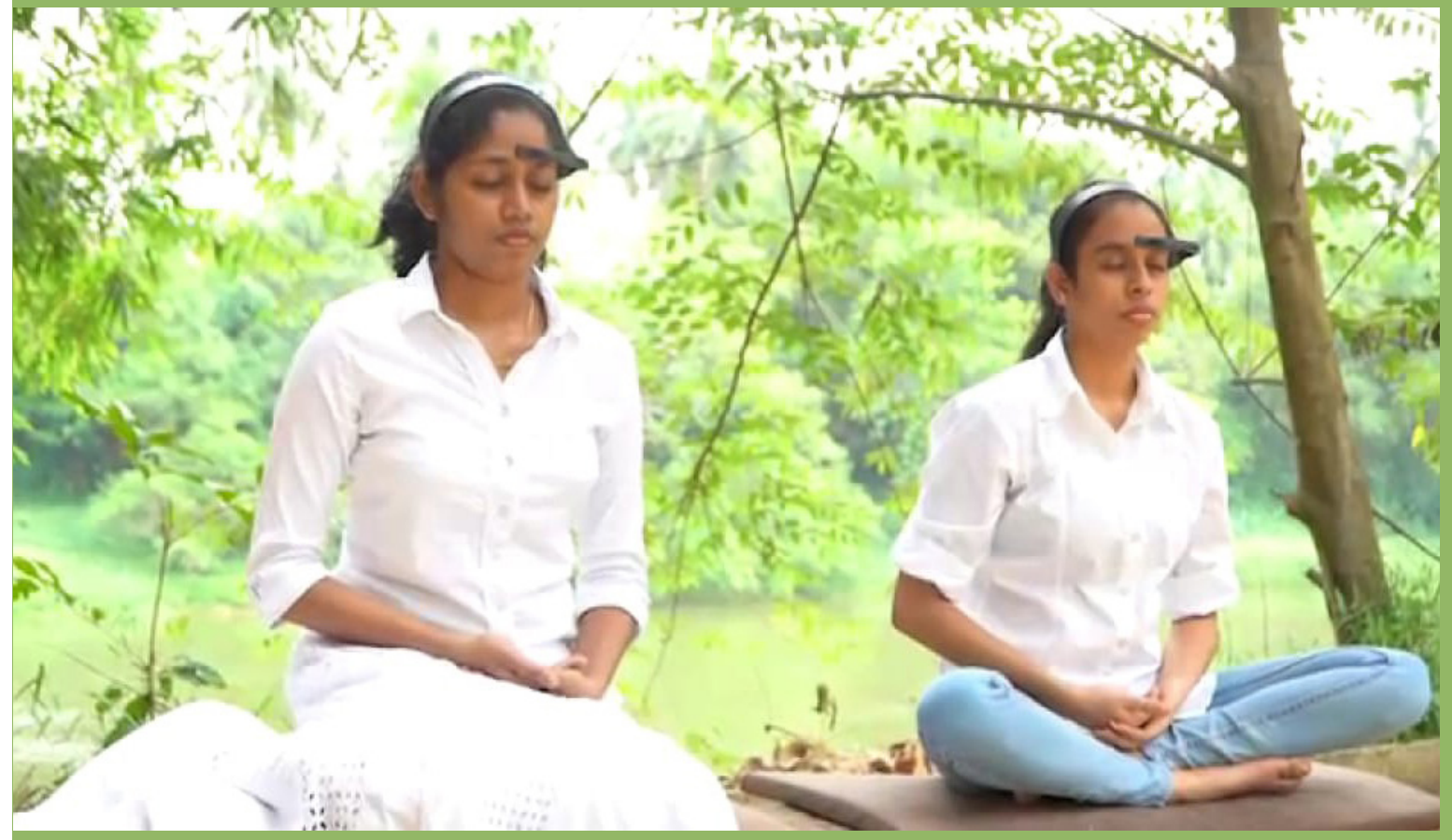

Power of understanding is a rewarding cognitive capacity required by all of us from early childhood to the highest level of intellectual settings. Among other things, the concept of understanding plays a vital role in education. When I was a second-year undergraduate, I was so curious to know why some of my colleagues could understand subjects much faster than the others, and this curiosity compelled me to research on how understanding manifests in our minds. My literature review revealed that the ultimate happiness/truth stated in Buddhism is a matter of understanding the world differently from the way we do it generally. Literature also showed that many people in Buddha's time understood the ultimate truth while listening to the discourse of the Buddha. Those who could not understand a matter then and there had to develop certain cognitive skills through various cognitive tasks such as further listening, discussing, thinking, and meditating. This is equally applicable to our educational settings as well because some students understand the subject matter during the lecture itself, while the others need to involve in additional reading activities, discussions, tutorial work, and so on. 


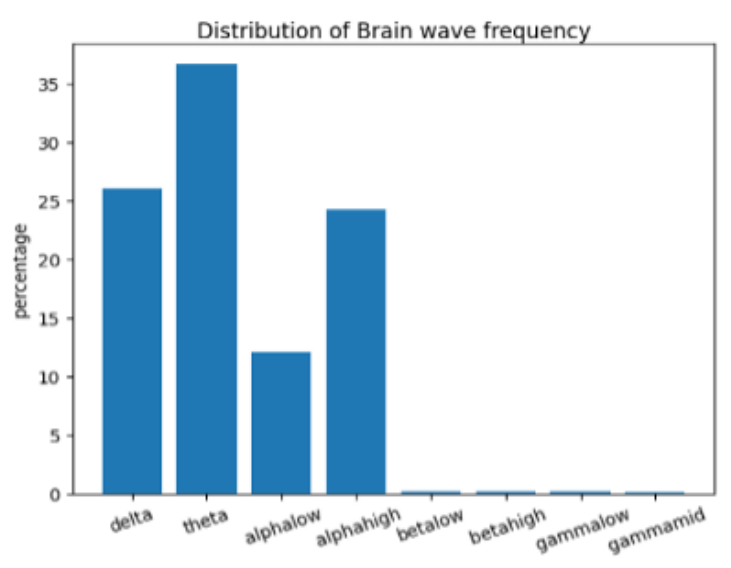

Figure 1-Higher mindfulness due to Higher Theta

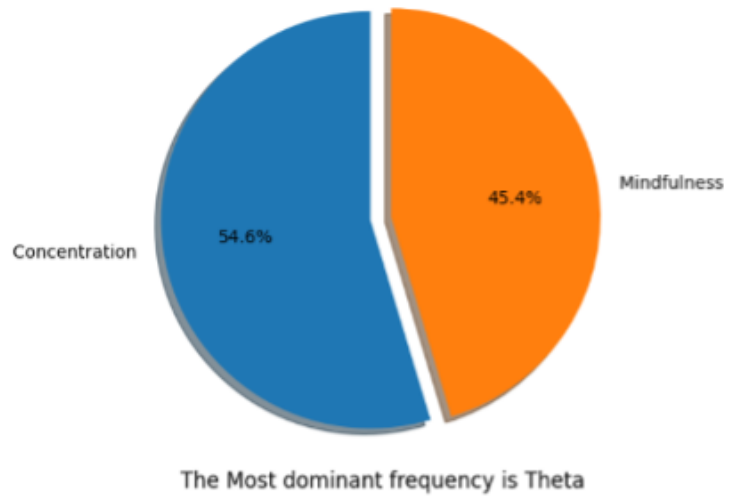

Figure 2 - Lesser Mindfulness when Theta is dominant?
Having read the discourse on Satipatthana [1], I discovered mindfulness as the foundation for understanding a subject matter. Mindfulness can be primarily seen as the present moment awareness of anything we experience in an impartial manner. Literature shows two major benefits of mindfulness (sampajano satima) to enable understanding. The first benefit of mindfulness is the effortless and swift recalling of the relevant knowledge at the correct time. The exciting second benefit of mindfulness is the ability to facilitate the understanding of new knowledge. Bringing the relevant subject matter to the surface effortlessly and solving previously unsolved problems are two phenomena that we experience in answering at examinations. If the mindfulness is low, the correct answer might surface after the examination. This finding reminds us that reading notes and books, answering past papers, doing practical work, and participating in discussions are techniques we used to improve mindfulness required for exams.

It should be noted that in addition to reading, discussing, working out past papers, etc. there are general exercises that help to cultivate mindfulness. Certain tasks such as medical surgeries, mathematical calculations, shooting, and walking on uneven surfaces etc. naturally require higher levels of mindfulness. Accordingly, people have in-

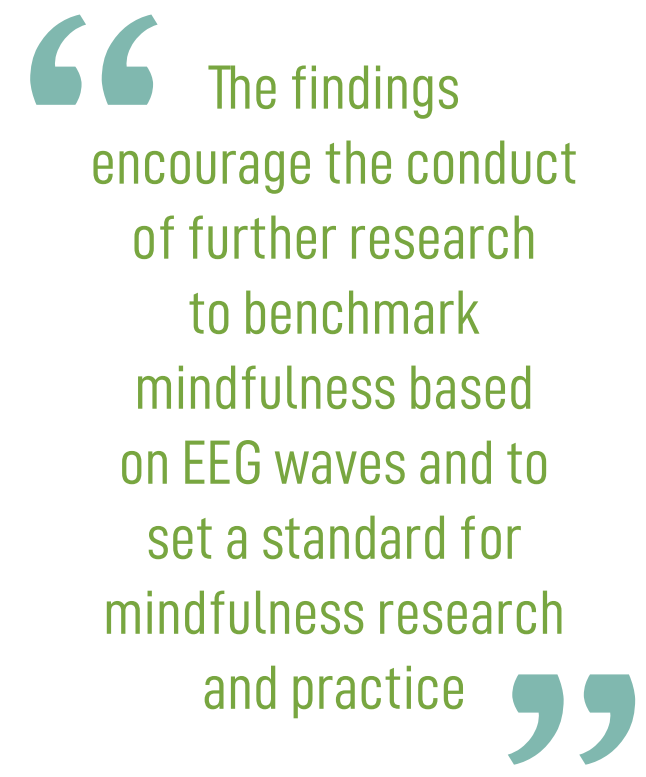

ness. In the recent past, a huge volume of research has been conducted on mindfulness interventions and to study the effect of mindfulness for various purposes including stress reduction, emotional balance, and enhancement of retention power and capacity to think [2]. Much of such research have been focused on realizing mindfulness through the analysis of EEG signals [3].

With this background, I have been engaged over the last decade, in investigating how the current 
research findings in mindfulness comply with the original Theravada literature on mindfulness. Undisputedly, Sri Lanka is an ideal place to conduct this kind of research due to the availability of access to original literature on mindfulness and long-established traditions/schools of the practice of mindfulness. In my research, I have used EEG technology to capture brain waves emitted from Fp1 location in the brain when people are involved in mindfulness practices. This experiment is based on mindfulness practitioners of Sati Pasala Foundation established under Nissarana Vanaya monastery at Meethirigala, Sri Lanka.

The first finding of the research is that although current literature predominantly talks of $\theta$ waves of the frequency range $4-8 \mathrm{~Hz}$ to describe mindful-

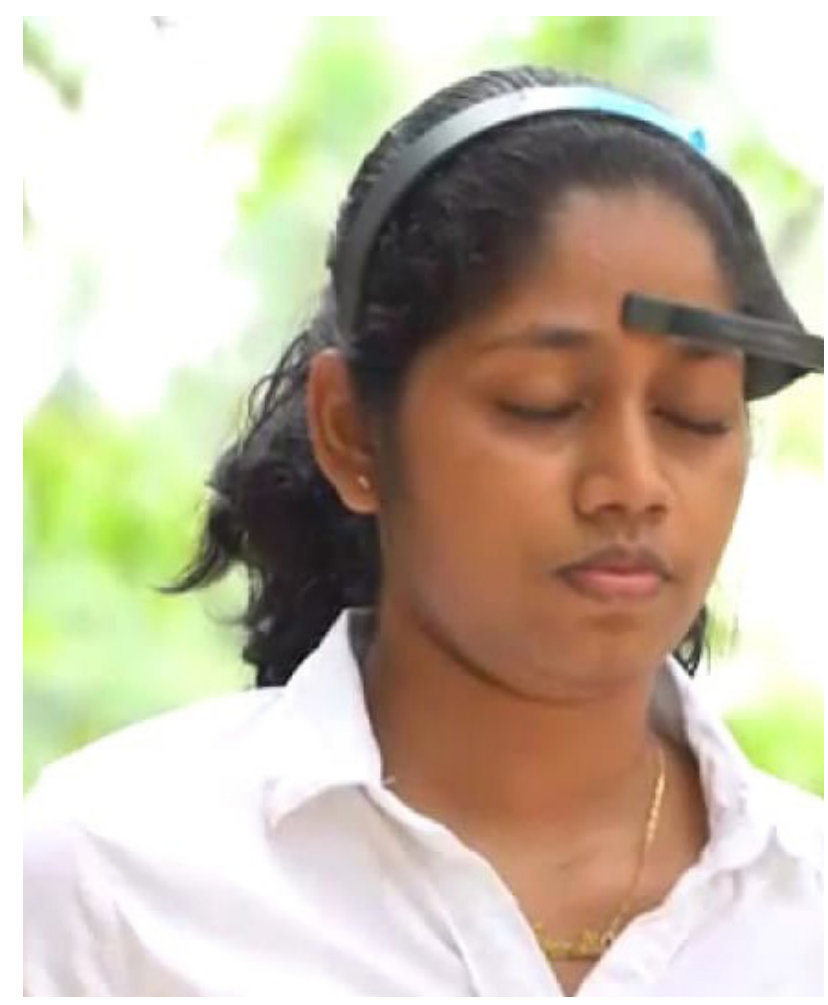

ness, our research shows in many instances, the other waves such as $\delta(1-4 \mathrm{~Hz})$ and $\alpha(8-13 \mathrm{~Hz})$, which are in the proximity of $\theta$, also contribute to determine the level of mindfulness. Fig. 1 shows the presence of $\delta, \alpha$ and $\theta$ together in a wave spectrum of a particular mindfulness session.

Thus, the findings shown in Fig. 1 and Fig. 2 are not comparable. The disparity might probably be due to the ignorance of the contribution of $\delta$ and $\alpha$ waves on determining the mindfulness. This anomaly is quite possible since many people have a confusion between mindfulness and concentration. Therefore, the discovery of a comprehensive brain wave spectrum for mindfulness remains a research challenge.

The above findings encourage further research to benchmark mindfulness based on EEG waves and to set a standard for mindfulness research and practice. It is planned to conduct a series of control experiments with experienced mindfulness-practitioners and to collect EEG signals. The experiment will be designed to practice mindfulness according to Theravada tradition of mindfulness. The findings of the further research would enable the novice mindfulness-practitioners to cultivate mindfulness effectively, and it would result in individuals getting the benefits of mindfulness training to energize cognitive skills such as understanding, analytical thinking, retention power, emotional balance, and social well-being.

\section{References}

[1] Analayo, Satipatthana: The Direct Path to Realization. Birmingham: Windhorse Publications, Birmingham, 2018.

[2] J. M. G. Williams and J. Kabat-Zinn, Mindfulness: diverse perspectives on its meaning, origins and applications. London, England; New York: Routledge, 2013.

[3] A. S. Karunananda, P. Goldin, and P. D. Talagala, "Examining Mindfulness in Education," Int. J. Mod. Educ. Comput. Sci., vol. 8, no. 12, pp. 23-30, Dec. 2016, doi: 10.5815/ijmecs.2016.12.04.

Article by

Asoka S. Karunananda

Department of Computational Mathematics, Faculty of Information Technology

University of Moratuwa, Sri Lanka. 